\title{
Energy-Efficient Relay Aided Ad Hoc Networks Using Iteratively Detected Irregular Convolutional Coded, Unity-Rate Coded and Space-Time Trellis Coded Transceivers
}

\author{
Jing Zuo, Hung Viet Nguyen, Soon Xin Ng and Lajos Hanzo \\ School of ECS, University of Southampton, SO17 1BJ, UK. \\ Tel: +44-23-8059 3125, Fax: +44-23-8059 4508 \\ Email: \{jz08r,hvn08r,sxn,lh\}@ecs.soton.ac.uk; http://www-mobile.ecs.soton.ac.uk
}

\begin{abstract}
The nodes of ad hoc networks are typically batterypowered, hence requiring the employment of energy-efficient schemes. Near-capacity coding schemes allow a single link to communicate using the lowest possible transmit power, while achieving a low Frame Error Ratio (FER). With the same motivation of conserving battery energy, numerous power-aware routing algorithms have been proposed for improving the overall multiuser network's energy-efficiency instead of improving a single link. Taking both these design factors into consideration, we employ Multi-Antenna aided Relays (MA-Rs) in the context of ad hoc networks, which use a three-stage concatenated transceiver constituted by an Irregular Convolutional Code, Unity-Rate Code and Space-Time Trellis Code (IrCC-URC-STTC) equipped with two antennas. All other mobiles are single-antenna assisted nodes and refrain from relaying, hence they dispense with STTC schemes and use a two-stage concatenated IrCC-URC coding scheme. It is confirmed that as expected, in a high-node-density scenario the average energy consumption per information bit and per node becomes about a factor two lower than that in the equivalent Single-Antenna Relay (SA-R) aided networks.
\end{abstract}

\section{INTRODUCTION}

Energy-efficient wireless network design has recently attracted wide-spread research attention. Diverse error-resilient Forward Error Correction (FEC) schemes were proposed in [1] for achieving a low Bit Error Ratio (BER) at near-capacity Signal-to-Noise Ratio (SNR) values. Therefore, the effective transmission range can be improved, when the required received signal power is reduced. A novel Distributed Concatenated Irregular Convolutional Coded, Unity-Rate Coded and Space-Time Trellis Coded (DC-IrCC-URC-STTC) scheme has been proposed for cooperative communications in [2]. Several Single-Antenna Relays (SA-Rs) were activated between the source and the destination. The relays roaming closest to their optimal locations were activated based on a novel technique relying on Extrinsic Information Transfer (EXIT) charts in conjunction with near-capacity code design principles.

Numerous power-aware routing protocols were proposed in [3] for improving the energy efficiency from a multiuser networking perspective. Moreover, cross-layer optimized power control has been widely exploited [4-8] for maintaining the required target-integrity at a low power in realistic propagation environments. A physical-layer-oriented routing protocol supported by sophisticated power control was proposed in [4] for a Line-Of-Sight (LOS) and shadow faded scenario, where the estimated end-to-end BER of a multi-hop path was used as the route selection metric. Furthermore, an adaptive relaying strategy switching between the Amplify-and-Forward (AF)

The research leading to these results has received funding from the European Union's Seventh Framework Programme (FP7/2007-2013) under gran agreement no 214625. The financial support of the China-UK Scholarship Council, and of the RC-UK under the auspices of the IU-ATC initiative is also gratefully acknowledged. and the Decode-and-Forward (DF) schemes was proposed in [5] for reducing both the energy consumption as well as the delay of the system. As a further design dilemma, the influence of the 'small number of long hops' versus the 'many short hops' philosophy on the energy consumption was studied in [6-8]. It was indicated in [6] that the 'small number of long hops' routing scheme was better than the 'many short hops' routing scheme provided that near-capacity coding strategies combined with a relatively short block length were employed, because a substantial SNR loss was exhibited by the 'many short hops' based routing scheme. Moreover, it was demonstrated in [7] that 'many short hops' perform well in energylimited scenarios relying on spatial reuse, even in the absence of interference cancellation, while using a 'small number of long hops' is more suitable for bandwidth-limited scenarios. Therefore, the routing algorithms should be carefully designed, when jointly considering both the achievable energy-efficiency and the attainable bandwidth-efficiency. The tradeoffs between energy- and bandwidth-efficiency were studied in [8], where it was found that at high end-to-end data rates the routes associated with fewer hops minimize the energy consumption, while at lower end-to-end data rates the routes having more hops mitigate it.

Against this backdrop, the novel contributions of this treatise are

- A near-capacity IRCC-URC-STTC transceiver is designed for multiple-antenna-relaying aided ad hoc networks, which is shown to reduce the network's total energy-consumption.

- This is achieved with the aid of sophisticated cross-layeroperation aided routing, where the routes are set up on the basis of using the lowest number of potentially longer hops, which become sufficiently reliable as a benefit of the powerful near-capacity IRCC-URC-STTC relaytransceivers.

To elaborate a litter further, we characterize the benefits of Multi-Antenna aided Relays (MA-Rs) in ad hoc networks in terms of the attainable energy efficiency. The MA-Rs are assisted by the Quadrature Phase-Shift Keying (QPSK) based IrCC-URC-STTC scheme proposed in [2], while the SA-Rs employ an 8-ary Phase-Shift Keying (8PSK) based IrCC-URC scheme. The introduction of the MA-Rs influences the route selection. More specifically, MA-Rs allow us to reduce the number of hops required as a benefit of their higher effective transmission range at a given transmit power compared to SA-Rs systems. Simulations are conducted for investigating the influence of both the number of MA-Rs and that of the node density on the energy consumption of the entire network. As the ultimate benchmark scheme, 
the Discrete-input Continuous-output Memoryless Channel's (DCMC) capacity [9] is considered.

The rest of the paper is organized as follows. In Section II, our IrCC-URC-STTC scheme and routing strategy are detailed. In Section III, various practical scenarios are analyzed, leading to our conclusions in Section IV.

\section{TheORETICAL AnAlysis}

\section{A. Near-Capacity Coding Schemes}

Irregular Convolutional Codes (IrCCs) [10] are constituted by a family of convolutional codes, which use different coderates for encoding the 'appropriately selected fractions' of the input stream. These IrCCs are employed as the outer code of our serial-concatenated transceiver scheme, since they are capable of approaching the channel capacity $[10,11]$. The 'appropriately selected fractions' are determined using the procedures proposed in [12] and detailed in [13] by exploiting the fact that a low BER may be achieved by the $\mathrm{IrCC}$ at SNRs near the capacity, when its EXIT-chart exhibits an open but narrow tunnel. Furthermore, as also detailed in [13], for the sake of guaranteeing a low BER and low Frame Error Ratio (FER), an infinite impulse response Unity-Rate Code (URC) should be employed as an intermediate code in order to ensure that the three-stage concatenated scheme indeed becomes capable of reaching the $(1,1)$ point of perfect convergence to a low BER in the EXIT chart [12]. The SpaceTime Trellis Code (STTC) employed is a Multi-Input MultiOutput (MIMO) arrangement, which is capable of achieving both a coding gain and a spatial diversity gain [13]. As a result, the IrCC-URC-STTC coding scheme advocated is capable of approaching the DCMC capacity of the SA-R aided cooperative system [2]. In this paper, we replace the pair of SA-Rs designed for the cooperative system of [2] with a single MA-R and demonstrate that the IrCC-URC-STTC scheme is capable of approaching the DCMC link's capacity derived for MA-R aided MIMO systems.

We assume that each MA-R is equipped with two antennas. If more than one MA-R exist in the multi-hop ad hoc network considered, then four different types of links may appear. Specifically, we have the SA-R to SA-R, SA-R to MA-R, MA$\mathrm{R}$ to SA-R and finally the MA-R to MA-R links. All the MARs employ the above-mentioned QPSK-assisted IrCC-URCSTTC scheme, while all the SA-Rs employ the 8PSK-assisted IrCC-URC scheme.

First, we analyze the attainable FER performance of all the four links, assuming that they are capable of reaching the DCMC capacity of the uncorrelated Rayleigh fading channel [9]. The Single-Input Single-Output (SISO) DCMC capacity is given by [14]:

$$
\begin{aligned}
C_{D C M C} & =\log _{2}(M) \\
& -\frac{1}{M} \sum_{m=1}^{m=M} E\left(\log _{2} \sum_{z=1}^{z=M} \exp \left(\psi_{m, z}\right) \mid X_{m}\right),
\end{aligned}
$$

where the expectation term in Eq. (1) is computed by employing the Monte Carlo averaging method. More specifically, $M$ is the number of modulation levels and $E\left(A \mid X_{m}\right)$ is the expectation of $A$ conditioned on $X_{m}$, while $\psi_{m, z}$ is given by

$$
\psi_{m, z}=\frac{-\left|h\left(X_{m}-X_{z}\right)+n_{m}\right|^{2}+\left|n_{z}\right|^{2}}{N_{0}} .
$$

Hence we can readily find the corresponding receive SNR value $\left.S N R_{r}\right|_{B P S}$ for a given Bits Per Symbol (BPS) throughput from the DCMC capacity curve computed using Eq. (1). The calculation of the DCMC capacity of the Single-Input Multi-Output (SIMO), Multi-Input Single-Output (MISO) and Multi-Input Multi-Output (MIMO) links can also be found in [14]. If the $\left.S N R_{r}\right|_{B P S}$ is higher than the SNR threshold of the corresponding capacity curve, the FER tends to 0, otherwise it tends to 1 . The FER performance of all the four links is portrayed in Fig. 1.

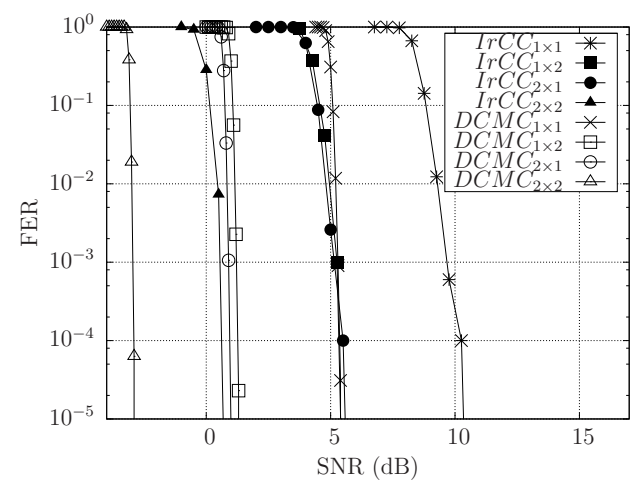

Fig. 1. FER performance of the four types of links at the frame length of 1500 bits of the uncorrelated Rayleigh fading channel evaluated from Eq. (1), ' $T \times R$ ' means $T$ transmit antennas and $R$ receive antennas.

The inverse second-power free-space path loss law and an uncorrelated Rayleigh fading channel model are considered in this paper. The received signal power $p_{r}$ can be formulated as [15]

$$
p_{r}=p_{t}\left(\frac{\sqrt{G_{l}} \lambda}{4 \pi d}\right)^{2} E\left[\|\mathbf{H}\|^{2}\right],
$$

where $p_{t}$ is the transmitted power, $\sqrt{G_{l}}$ is the product of the transmit and receive antenna gains in the LOS direction, $\mathbf{H}$ is the matrix of Channel Impulse Responses (CIRs), $d$ is the average distance from the transmitter and $\lambda$ is the carrier's wavelength. We assume $\sqrt{G_{l}}=1, E\left[\|\mathbf{H}\|^{2}\right]=1$ and $\lambda=$ $c / f$, where $c$ is the speed of light in vacuum and $f$ is the carrier frequency. Hence, we have

$$
d=\sqrt{\frac{p_{t}}{p_{r}}} \frac{\lambda}{4 \pi} .
$$

The Signal-to-noise ratio $S N R(d B)$ is calculated as

$$
S N R=10 \log _{10}\left(\frac{p_{r}}{N_{0}}\right)[d B],
$$

where $N_{0}$ is the thermal noise power and $N_{0}(d B)=$ $10 \log _{10} N_{0}$. Then received signal power $p_{r}$ may be expressed as

$$
p_{r}=10 \frac{S N R+N_{0}(d B)}{10} .
$$

Hence, given the above-mentioned parameters, the average distance from the transmitter, where the SNR requirement may 
'just' be satisfied, is given by

$$
d=\frac{\sqrt{p_{t}} \lambda}{4 \pi 10} .
$$

Naturally, if we fix the value of $p_{t}$ and $N_{0}(d B)$, then it may be readily seen how the adequately 'illuminated' distance, where the required target-FER may be maintained, will vary as a function of the SNR value. As seen from Fig. 1, the maximum adequately covered communication distance from MA-R to MA-R is the highest, while that from SA-R to SA$\mathrm{R}$ is the lowest. Conversely, if $p_{t}$ and $d$ are fixed, then the FER is the lowest for the MA-R to MA-R link, while it is the highest for the SA-R to SA-R link.

\section{B. Routing Algorithms}

It was shown in [2] that the IrCC-URC-STTC scheme is capable of operating near the link's capacity, hence a substantial power saving may be attained. When this scheme is employed by the MA-Rs of the ad hoc network considered, the different error correction capability of the four different types of links will influence the routing strategy. Fig. 2 provides an example on how the routing strategy is influenced, as detailed below.

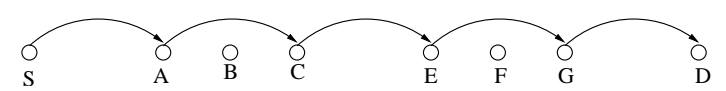

(a)

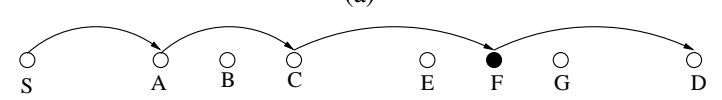

(b)

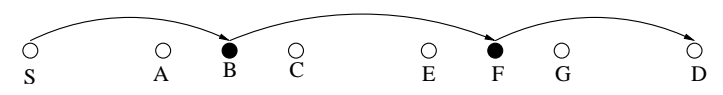

(c)

Fig. 2. The influence of MA-Rs on the routing strategy: (a) $h=5$ hops without MA-Rs; (b) $h=4$ with 1 MA-R at point $F$; (c) $h=3$ with 2 MA-Rs at points $B$ and $F$.

As seen from Fig. 2, the network consists of $N=8$ nodes, where $S$ is the source and $D$ is the destination. In Fig. 2(a), all nodes are equipped with a single antenna, hence all links are SA-R to SA-R links, which yields $h=5$ hops from $S$ to $D$. A single MA-R is employed at point $F$ in Fig. 2(b), where the packets arriving at node $C$ are directly transmitted to node $F$. Then, node $F$ will relay its received packets further to the destination $D$. More specifically, the $C$-to- $F$ link is a SA-R to MA-R link, while the $F$-to- $D$ link is an MA-R to $D$ link, where the $F-D$ distance is higher than that between the single-antenna nodes of Fig. 2(a). Consequently, the number of hops from $S$ to $D$ is decreased to $h=4$. In Fig. 2(c), two MA-Rs, namely $B$ and $F$, are employed. The number of hops is further decreased to $h=3$ as a benefit of using MA-Rs for nodes $B$ and $F$.

This paper employs the DYnamic Manet On-demand (DYMO) [16] routing protocol in the network layer, which combines most of the benefits of the Ad-hoc On-demand Distance Vector (AODV) [17] and Dynamic Source Routing (DSR) [18] protocols. The DYMO routing protocol always opts for the specific route having the lowest number of hops to the destination. When employing the MA-R aided IrCC-URCSTTC scheme, we will demonstrate that the route selected may be expected to have a further reduced number of hops. The DYMO routing protocol is constituted by two main stages, namely the route discovery and route maintenance. During the route discovery, the Route REQuest (RREQ) and the Route REPly (RREP) packets are used for identifying a route from the source to the destination. By contrast, during the route maintenance phase, a Route ERRor (RERR) packet is returned to the source, when a broken link is detected. We assume that every node has the same transmit power of $p_{t}$. Consequently, the sum of the energy dissipated by all nodes in the network is given by

$$
\begin{aligned}
E_{T}= & \sum E_{\text {Route_Discovery }} \\
& +\sum\left(p_{t} \frac{n_{\text {data }}}{r_{\text {data }}}\right)+\sum\left(p_{t} \frac{n_{\text {wlan }-A C K}}{r_{\text {wlan }-A C K}}\right) \\
& +\sum E_{\text {Route_Maintenance }},
\end{aligned}
$$

where $n_{\text {data }}$ is the length of every packet arriving from the Medium Access Control (MAC) layer, except for the ACKnowledgment ${ }^{1}$ (wlan-ACK) packet. The length of the wlan-ACK packet is $n_{\text {wlan-ACK }}$. The information bit rate for transmitting data and wlan-ACK packet is denoted by $r_{\text {data }}$ and $r_{\text {wlan-ACK }}$, respectively. We assume that the wlanACK packet is protected by powerful, low-rate coding and modulation schemes and seldom has erroneous bits, hence $r_{\text {wlan-ACK }}$ is different from $r_{\text {data }}$. Furthermore, on one hand, $\sum E_{\text {Route_Discovery }}$ denotes the sum of energy consumed by the RREQ, the RREP and the wlan-ACK packets during the route discovery phase. On the other hand, $\sum E_{\text {Route_Maintenance }}$ includes all the energy except for the energy dissipated by the data packets and the corresponding wlan-ACK packets during the route maintenance phase. Only the transmit power is considered in Eq. (8).

Furthermore, let $B P S_{M A}$ and $B P S_{S A}$ denote the bits per symbol throughput of the MA and SA nodes, respectively. The IrCC, URC and STTC component codes have code rates of $0.5,1.0$ and 1.0, respectively. Since QPSK is used at the MA nodes and 8PSK at the SA nodes, we have $B P S_{M A}=$ $2 B P S_{S A} / 3$ and then $r_{M A}=2 r_{S A} / 3$, where $r_{M A}$ and $r_{S A}$ are the bit rate at the MA-Rs and SA-Rs, respectively. Hence, Eq. (8) may be simplified as:

$$
\begin{aligned}
E_{T} & =\sum E_{\text {Route_Discovery }} \\
& +\frac{3 p_{t} n_{\text {data }}-1}{2 r_{S A}} r_{m a}+\left(\frac{p_{t} n_{\text {data }}}{r_{S A}}+\frac{p_{t} n_{\text {wlan }-A C K}}{r_{\text {wlan-ACK }}}\right) h \\
& +\sum E_{\text {Route_Maintenance }},
\end{aligned}
$$

where again $h$ is the number of hops of the selected route. First, we assume that $p_{t}, n_{\text {data }}, n_{\text {wlan }-A C K}$ and $r_{\text {wlan }-A C K}$ are constant values. Then we introduce the short-hand of $A=$ $\frac{3 p_{t} n_{\text {data }}-1}{2 r_{S A}}$ and $B=\left(\frac{p_{t} n_{\text {data }}}{r_{S A}}+\frac{p_{t} n_{\text {wlan }-A C K}}{r_{\text {wlan-ACK }}}\right)$. Furthermore, $\sum E_{\text {Route_Discovery }}$ is replaced by $f_{1}\left(\rho, r_{m a}, \vec{v}\right)$,

\footnotetext{
${ }^{1}$ The Acknowledgement packet is the one, which is returned to the transmitter as the acknowledgement of the correctly received data in the MAC layer, hence we refer to it as wlan-ACK in this paper, where 'wlan-ACK' means the ACK packet employed in the IEEE802.11 standard. We assume that there is no Request-To-Send (RTS)/Clear-To-Send (CTS) mechanism employed.
} 
and $\sum E_{\text {Route_Maintenance }}$ is replaced by $f_{2}\left(\rho, r_{m a}, \vec{v}\right)$, because they are influenced by the node density $\rho$, the number of MA-Rs $r_{m a}$ and the mobile speed $\vec{v}$. Then, Eq. (9) can be written as:

$$
E_{T}=f_{1}\left(\rho, r_{m a}, \vec{v}\right)+A r_{m a}+B h+f_{2}\left(\rho, r_{m a}, \vec{v}\right) .
$$

We further normalize $E_{T}$ by the overall number of bits $L$ received in the application layer of the destination and the number of nodes $n$ of the entire network, where $n$ is related to $\rho$ and $L$ is related to $n_{\text {data }}$. Finally, the overall energy consumption $E_{N T}$ of the entire network can be expressed as:

$$
E_{N T}=\frac{E_{T}}{n L}=F\left(\rho, r_{m a}, \vec{v}, h, B P S_{S A}, L\right) .
$$

\section{PERformance Study}

As seen from Eq. (11), the overall energy consumption of the entire network is dependent on numerous parameters, such as the node density, the number of MA-Rs, the mobile speed, the number of hops of the selected route, the BPS throughput and the amount of bits received in the application layer of the destination. In order to reduce the dimensionality of our investigations, we fix some of the parameters when characterizing the benefits of MA-Rs on the node's achievable transmission range and FER performance. More explicitly, we will mainly focus our attention on the relationship between the number of MA-Rs and the energy consumption, as well as that between the node density and the energy consumption. The simulation configurations considered are as follows:

- The application layer generates $P=5$ packets per second and the length of each packet is $L_{p}=504$ bits;

- The User Datagram Protocol (UDP) [19] is employed in the transport layer;

- The DYMO [16] routing protocol is chosen in the network layer;

- IEEE802.11b [20] is used in the MAC layer;

- The IrCC-URC-8PSK [2] and IrCC-URC-STTCQPSK [2] schemes are employed in the physical layer for SA and MA nodes, respectively;

- Every node transmits at $1 \mathrm{~mW}$ power.

\section{A. Energy Consumption versus The Number of MA-Rs}

Assuming that the node density $\rho$, the mobile speed $\vec{v}$, the BPS throughput $B P S_{S A}$ and the number of bits $L$ received in the application layer of the destination are constant, Eq. (11) can be simplified to:

$$
E_{N T}=F\left(r_{m a}, h\right) .
$$

Four scenarios are considered in order to study the relationship between the number of MA-Rs and the energy consumption, where $n=60$ stationary nodes are randomly located in a $500 \mathrm{~m} \times 500 \mathrm{~m}$ field, hence the node density is $\rho=240$ nodes per square kilometers. The source and the destination are located in the position $(0,0)$ and $(499,499)$, respectively. We increase the number of MA-Rs ${ }^{2}$ from $r_{m a}=0$ to $r_{m a}=60$ in steps of 5 . The receiver's sensitivity [21] is set to -85

\footnotetext{
${ }^{2}$ The number of MA-Rs also includes the source and the destination. Again, we denote the multi-antenna aided nodes as MA-Rs and single-antenna nodes as SA-Rs.
}

$\mathrm{dBm}$. Any signal received at a power lower than the noise sensitivity threshold is deemed to be undetectable. The power required is quantified for the IrCC-URC-STTC scenario ${ }^{3}$ and benchmarked against the DCMC capacity at the thermal noise levels of $-92 \mathrm{dBm}$ and $-86 \mathrm{dBm}$. We also quantify the Packet Loss Ratio (PLR) and the network control load in order to characterize the performance of the entire network. We define the network control load $^{4}$ as the number of routing control bits transmitted in the entire network divided by the number of data bits delivered to the destination [22].

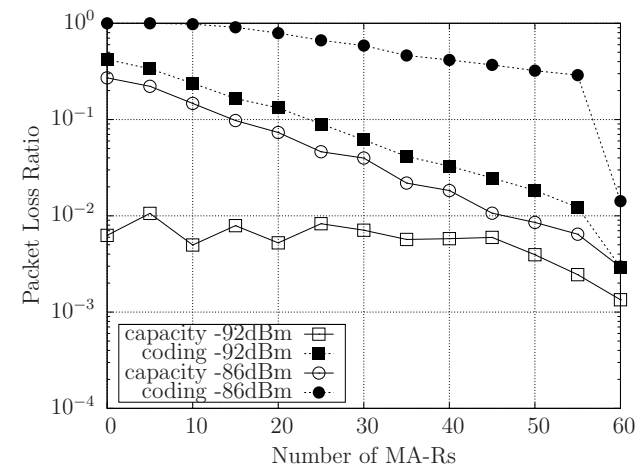

Fig. 3. PLR versus the number of MA-Rs

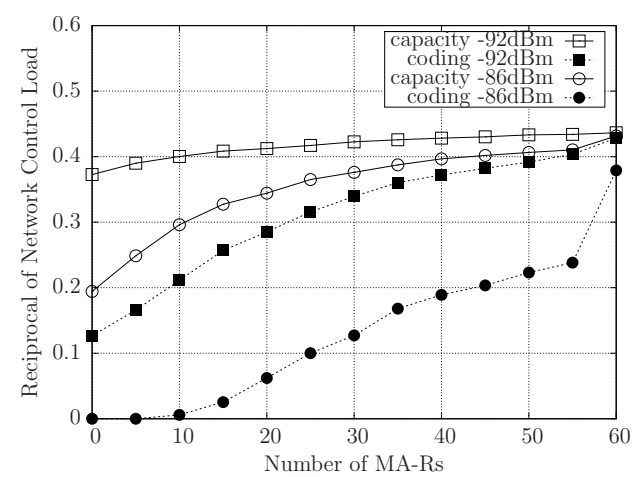

Fig. 4. Reciprocal of the network control load versus the number of MA-Rs

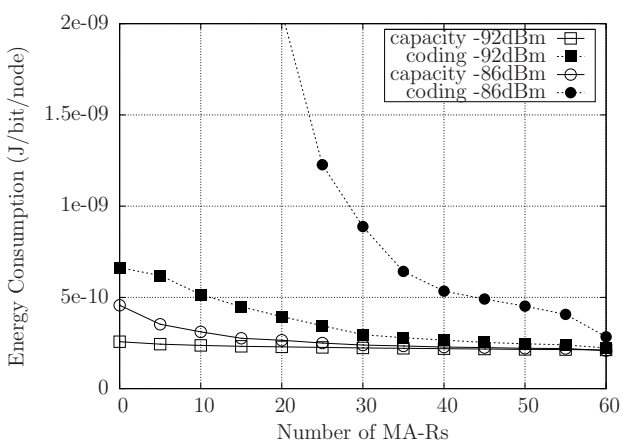

Fig. 5. Energy consumption versus the number of MA-Rs

As seen from Fig. 3, Fig. 4 and Fig. 5, the PLR, the network control load and the energy consumption of the IrCC-URCSTTC scheme and of the benchmark scheme decrease upon increasing the number of MA-Rs. As mentioned in Section II-A,

${ }^{3}$ In the IrCC-URC-STTC scenario we assume that the network consists of the QPSK-assisted IrCC-URC-STTC aided MA-Rs and the 8PSK-assisted IrCC-URC aided SA-Rs.

${ }^{4}$ In order to avoid the appearance of an infinite network control load, when no data are correctly received in the network layer, the reciprocal of the network control load is used instead of the control load itself. 
a low FER and a relatively high transmission range i.e. coverage area may be ensured by using the IrCC-URC-STTC scheme advocated. Furthermore, as justified in Section II-B, the specific routes having the lowest number of hops tend to be activated in the MA-Rs aided network considered. Therefore, as expected, the PLR decreases, when the number of MARs increases. Moreover, having a high physical-layer FER results in an increased number of retransmissions and hence may trigger route re-discovery, which results in more control packets being transmitted. Hence, more energy per payload bit is required for successfully delivering the source data to the destination, as demonstrated in Fig. 5.

\section{B. Energy Consumption versus Node Density}

Assuming that the number of MA-Rs $r_{m a}$, the mobile speed $\vec{v}$, the BPS throughput $B P S_{S A}$ and the number of bits $L$ received in the application layer of the destination are constant, Eq. (11) can be simplified to:

$$
E_{N T}=F(n, h) .
$$

Four scenarios are considered in order to study the relationship between the node density and the overall energy consumption of the entire network, where all the nodes are stationary and randomly located in a $500 \mathrm{~m} \times 500 \mathrm{~m}$ field. The source and the destination are located in the position $(0,0)$ and $(499,499)$, respectively. We increase the node density from $\rho=80$ to $\rho=400$ in steps of 80 . The receiver's sensitivity is set to $-85 \mathrm{dBm}$ and the thermal noise is set to $-92 \mathrm{dBm}$. The energy consumption, the PLR and the network control load are quantified for both the IrCC-URC-STTC scheme and the benchmarker based on the DCMC capacity when $r_{m a}=0$, 5,10 and all nodes are equipped with two antennas in the ad hoc network considered.

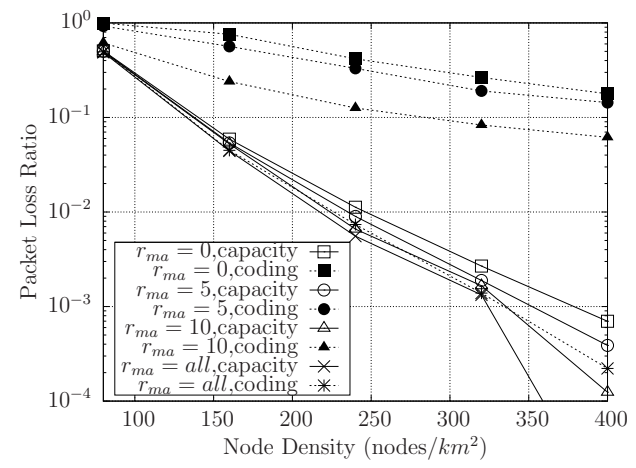

Fig. 6. PLR versus node density.

Fig. 6 shows that the PLR decreases upon increasing the node density. A high node density implies having short average distances between the pairs of nodes, hence a low PLR would be achieved as a benefit of having a high receive SNR. However, a high node density is also expected to impose a high interference on each node, which is expected to reduce the Signal-to-Interference Ratio (SIR). To explicitly characterize the underlying trends, Fig. 7 shows that the network control load is reduced for the capacity-based bechmarker - i.e. its reciprocal is increased - upon increasing the node density to $\rho=160$, since the benefits of the increased received signal power outweigh the detrimental effects of the degraded SIR.

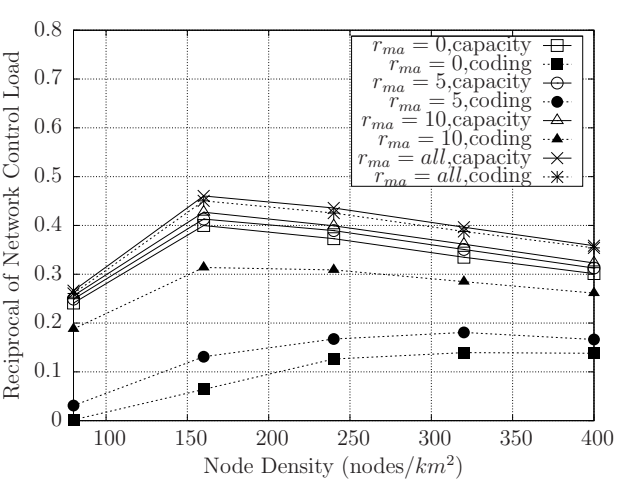

Fig. 7. Reciprocal of the network control load versus node density.



Fig. 8. Energy consumption versus node density.

This trend was reversed above $\rho=160$ for the DCMCcapacity-based benchmarker. By contrast, for the proposed IrCC-URC-STTC scheme, we observe that in the presence of $r_{m a}=0$ or 5 MA-Rs the network control load tends to gently decrease upon increasing the overall node density. Finally, the overall energy consumption expressed in Joule/bit/node is portrayed in Fig. 8. The benchmarker based on the DCMC capacity could be deemed to represent the so-called 'perfect' coding scheme, which is capable of communicating at the capacity limit. Therefore, it may be readily seen from Fig. 8 that near-capacity coding schemes benefit more substantially in high-node-density scenarios, when aiming for a high energy efficiency.

\section{CONCLUSIONS}

A near-capacity three-stage concatenated IrCC-URC-STTC relay-transceiver equipped with two antennas was proposed for the ad hoc network considered, since it was capable of achieving a low FER at a low transmit power. The high effective transmission range of the IrCC-URC-STTC aided MA-Rs allows us to use cross-layer optimization for activating routes having the lowest number of longer hops. The simulation results confirm that the energy consumption per bit and per node in the MA-R aided network becomes lower than that in the equivalent SA-R aided network, especially in high-nodedensity scenarios.

\section{REFERENCES}

[1] M. C. Vuran and I. F. Akyildiz, "Error control in wireless sensor networks: A cross layer analysis," IEEE/ACM Transactions on Networking, vol. 17, no. 4, pp. 1186 -1199, Aug. 2009. 
[2] H. V. Nguyen, S. X. Ng, and L. Hanzo, "Distributed three-stage concatenated irregular convolutional, unity-rate and space-time trellis coding for single-antenna aided cooperative communications," in Proc. 72nd IEEE on Vehicular Technology Conference VTC 2010-Fall, Sept. 2010.

[3] J. G. Li, D. Cordes, and J. Y. Zhang, "Power-aware routing protocols in ad hoc wireless networks," IEEE Wireless Communications, vol. 12 no. 6 , pp. $69-81$, Dec. 2005 .

[4] G. Ferrari, S. A. Malvassori, and O. K. Tonguz, "On physical layeroriented routing with power control in ad hoc wireless networks," IET Communications, vol. 2, no. 2, pp. $306-319$, Feb. 2008.

[5] J. C. Fricke, M. M. Butt, and P. A. Hoeher, "Quality-oriented adaptive forwarding for wireless relaying," IEEE Communications Letters, vol. 12, no. 3, pp. $200-202$, March 2008.

[6] M. Haenggi and D. Puccinelli, "Routing in ad hoc networks: a case for long hops," IEEE Communications Magazine, vol. 43, no. 10, pp. 93 101, Oct. 2005.

[7] M. Sikora, J. N. Laneman, M. Haenggi, D. J. Costello, and T. E. Fuja, "Bandwidth- and power-efficient routing in linear wireless networks," IEEE Transactions on Information Theory, vol. 52, no. 6, pp. 2624 2633, June 2006

[8] C. Bae and W. E. Stark, "End-to-end energy and bandwidth tradeoff in multihop wireless networks," IEEE Transactions on Information Theory, vol. 55, no. 9, pp. $4051-4066$, Sept. 2009.

[9] L. Hanzo, S. X. Ng, T. Keller, and W. Webb, Quadrature Amplitude Modulation: From Basics to Adaptive Trellis-Coded, Turbo-Equalised and Space-Time Coded OFDM, CDMA and MC-CDMA Systems. John Wiley and Sons, Ltd, 2004.

[10] M. Tucher and J. Hagenauer, "Exit charts of irregular codes," in Proc. 36 th Annual Conference on Information Sciences and Systems 2002 March 2002.

[11] M. Tuchler, "Design of serially concatenated systems depending on the block length," IEEE Transactions on Communications, vol. 52, no. 2, pp. $209-218$, Feb. 2004

[12] S. X. Ng, J. Wang, M. X. Tao, L. L. Yang, and L. Hanzo, "Iteratively decoded variable length space-time coded modulation: Code construction and convergence analysis," IEEE Transactions on Wireless Communications, vol. 6, no. 5, pp. 1953 -1963, May. 2007.

[13] L. Hanzo, O. Alamri, M. E. Hajjar, and N. Wu, Near-Capacity MultiFunctional MIMO Systems. New York, USA: John Wiley and Sons, 2009.

[14] S. X. Ng and L. Hanzo, "On the MIMO channel capacity of multidimensional signal sets," IEEE Transactions on Vehicular Technology, vol. 55, no. 2, pp. 528 - 536, March 2006.

[15] A. Goldsmith, Wireless Communications. Cambridge University Press, 2005.

[16] Dynamic MANET On-demand (DYMO) Routing draft-ietf-manet-dymo21, http://tools.ietf.org/html/draft-ietf-manet-dymo.

[17] Ad hoc On-Demand Distance Vector (AODV) Routing, http://tools.ietf.org/html/rfc3561.

[18] The Dynamic Source Routing Protocol (DSR) for Mobile Ad Hoc Networks for IPv4, http://tools.ietf.org/html/rfc4728.

[19] User Datagram Protocol, http://tools.ietf.org/html/rfc768.

[20] Corrigenda to 802.11b-1999 Higher Speed Physical Layer Extension in the $2.4 \mathrm{GHz}$ band, http://standards.ieee.org/getieee802/download/802.11b1999 Cor12001.pdf.

[21] J. Zuo, S. X. Ng, and L. Hanzo, "Fuzzy logic aided dynamic source routing in cross-layer operation assisted ad hoc networks," in Proc. 72nd IEEE on Vehicular Technology Conference VTC 2010-Fall, Sept. 2010.

[22] C. E. Perkins, E. M. Royer, S. R. Das, and M. K. Marina, "Performance comparison of two on-demand routing protocols for ad hoc networks," IEEE Personal Communications, vol. 8, no. 1, pp. 16 -28, Feb. 2001. 